\title{
Neurodevelopmental Outcomes for Infants Treated with Whole Body Cooling After Hypoxic- Ischemic Encephalopathy with and without Clinical Seizures: Retrospective Chart Review
}

\author{
Childres J* and Shaffer-Hudkins E \\ Department of Pediatrics, University of South Florida, USA
}

*Corresponding author: Jillian L Childres, Pediatric School Psychologist, Assistant Professor, University of South Florida, USA

Submission: 眥 January 05, 2018; Published: 無 July 19, 2018

\begin{abstract}
Introduction: This study described the developmental outcomes of infants who were diagnosed with Hypoxic-Ischemic Encephalopathy (HIE) after birth and were treated with whole body therapeutic hypothermia. Despite multiple studies on the efficiency and safety of therapeutic hypothermia for newborns with HIE little research is available specifically comparing developmental outcomes of infants with and without clinical seizure activity who have received this treatment.
\end{abstract}

Methods: We retrospectively analyzed cases of HIE in a Level 3 NICU who were treated with therapeutic hypothermia according to the hospital's standard procedures. Demographic data and test scores were analyzed for the entire group of infants and scores were compared for the group of infants with versus without neonatal seizures.

Results: The study consisted of data collected from the medical records of 24 surviving infants. Demographic data were comparable for the seizures and no seizures groups for all variables analyzed except for maternal age (significantly younger mean age of mothers in the group of infants with seizures than without seizures). The group of infants without neonatal seizures had higher mean scores across all developmental domains than the group of infants with neonatal seizures; however, clinical significance in these differences were only achieved in two score areas.

Conclusion: Results of this study indicated that the majority of the infants had overall developmental scores that indicate Moderate to Severe Developmental Delays despite receiving the whole body cooling treatment. Specific comparisons between the group of infants with and without seizures who received this treatment indicated better developmental outcomes for infants without seizures than for those infants who had seizures, though statistically significant differences were found in one specific sub domain and in the overall Total Developmental Quotient. More of the infants in the no seizure group had developmental scores in the Average range than did infants in the seizures group.

Keywords: Neonatal, Hypoxic-Ischemic Encephalopathy (HIE); Therapeutic hypothermia; Seizures development; Outcomes

Abbreviations: HIE: Hypoxic-Ischemic Encephalopathy; NICU: Neonatal Intensive Care Unit; IDEA: Individuals with Disabilities Education Act; BDI-II: Battelle Developmental Inventory; EEG: Electroencephalogram; PA: Perinatal Asphyxia

\section{Introduction}

The study described the medical, neurological, and developmental outcomes of infants who were diagnosed with Hypoxic-Ischemic Encephalopathy (HIE) after birth and were treated with total body hypothermia (body cooling). The study provides data on the efficacy of total body cooling in this specific cohort of infants. The data were obtained by a retrospective review of medical and developmental records of infants born during a twoyear period who were treated in the Neonatal Intensive Care Unit (NICU) at a hospital in Central Florida.
In 2009, the hospital's Level 3 NICU adopted as standard procedure the use of therapeutic hypothermia as a neuroprotective treatment for full-term infants diagnosed with HIE secondary to perinatal asphyxia (PA). In the first two years of implementation of this new protocol, 29 infants received the cooling procedure at this site. Despite multiple studies on the efficiency and safety of therapeutic hypothermia for newborns with HIE [1], there have been few studies specifically investigating the effects of clinical seizure activity before and after the cooling procedure on infant 
and early childhood outcomes. These early studies looking at the effects of seizures on outcomes were primarily focused on comparing outcomes between infants in the control (non-cooled) group and infants in the treatment (cooled) groups [2]. Little research is available specifically comparing outcomes of infants with and without clinical seizure activity who have been treated with therapeutic hypothermia.

The primary goal of this research study was to investigate the neurodevelopmental outcomes of therapeutic hypothermia as a neuroprotective treatment for HIE as implemented at a specific hospital site and to explore the possibility of differential outcomes given the presence or absence of neonatal seizures. Data for this study was collected from birth records including data on the cooling process, neurological consultations, follow-up while in the NICU, and data collected by a state funded developmental early intervention program (IDEA Part C).

\section{Hypothesis}

a. Infants in the study will have comparable neurodevelopment to same-age, typically developing peers, as indicated by scores within the average or expected range on the Battelle Developmental Inventory, $2^{\text {nd }}$ Edition (BDI-II) (between -1 and +1 SD of the mean $=85-115$ ) at all assessment times

b. Infants who were diagnosed with neonatal seizures prior to discharge will demonstrate significantly lower scores on measures of neurodevelopmental functioning than infants without this diagnosis at all assessment times.

\section{Methods}

Approval to conduct the research was granted by the Florida Department of Health. This study utilized a retrospective clinical series (case series) design, by tracking patients with a known condition who were given similar treatments and collecting data by examining medical records for treatment and outcomes. A small sample size was anticipated because the focus of this project is on outcomes related to a specific medical diagnosis (HIE) in a restricted subset of the population (newborns meeting inclusion criteria for hypothermia as a neuroprotective treatment).

Subjects were secured from a list of newborns treated with therapeutic hypothermia in the NICU at one hospital during a twoyear time frame. Consent was secured from the infants' parents by mailing home a consent form and a cover letter as well as a phone call from one of the investigators explaining the research process. After securing consent, subjects were not contacted again, only data that was previously collected and stored in medical or developmental records was analyzed. We reviewed the electronic medical records for each of the subjects whose parents signed a consent form. Since the subjects varied by age based on date of birth, follow-up visits and assessments were reviewed as they became available in the medical records. Data collection was conducted on an ongoing basis and periodic reviews and summaries of available information were completed every 6 months.

Of the 29 babies treated with whole-body cooling within the two-year period, 3 infants died prior to discharge from the NICU, 1 infant was discharged to a skilled nursing facility, and 25 infants were discharged home. Medical records were not available for the 3 deceased infants, the 1 infant discharged to the skilled nursing facility, and 1 of the surviving infants who moved out of state. All preliminary data analyses were done with the sample of the 24 infants' available medical records. Of the 24 infants, 12 infants, records included diagnoses of seizures and 12 did not; 16 infants were rated as having "moderate/moderate to severe" encephalopathy and 8 were rated as having "severe" encephalopathy. Demographic characteristics were similar for subjects across both groups with the exception of the variable maternal age. Younger maternal ages were found in the seizures group $(M=25.7, S D=6.2)$ than in the no seizures group ( $\mathrm{M}=32.3, \mathrm{SD}=6.2) ; \mathrm{t}(22)=2.605$ $p=0.02$. Refer to Table 1 for a summary of selected maternal and infant characteristics.

Table 1: Maternal and infant characteristics at discharge.

\begin{tabular}{|c|c|c|c|c|}
\hline Characteristics & Overall $n=24$ & Seizures $n=12$ & No Seizures $n=12$ & p value \\
\hline Maternal age, range (mean), years & $17-43(29.0)$ & $17-37(25.7)$ & $23-43(32.3)$ & $.016 a$ \\
\hline Method of delivery & & & & $.117 \mathrm{~b}$ \\
\hline Emergency C-section, n (\%) & $14(58.3)$ & $8(66.7)$ & $6(50.0)$ & \\
\hline Vaginal, unassisted, n (\%) & $8(33.3)$ & $2(16.7)$ & $6(50.0)$ & \\
\hline Vaginal, vacuum assisted, n (\%) & $2(8.3)$ & $2(16.7)$ & $0(0.0)$ & \\
\hline Neonatal Gender & & & & $1.00 \mathrm{~b}$ \\
\hline Male, n (\%) & $12(50.0)$ & $6(50.0)$ & $6(50.0)$ & \\
\hline Female, n (\%) & $12(50.0)$ & $6(50.0)$ & $6(50.0)$ & \\
\hline Pregnancy Complications & & & & $.083 \mathrm{~b}$ \\
\hline No complications, n (\%) & $8(33.3)$ & $6(50.0)$ & $2(16.7)$ & \\
\hline Complications, n (\%) & $16(66.7)$ & $6(50.0)$ & $10(90.9)$ & \\
\hline Delivery Complications & & & & $.273 \mathrm{~b}$ \\
\hline No complications, n (\%) & $4(16.7)$ & $1(8.3)$ & $3(25.0)$ & \\
\hline
\end{tabular}




\begin{tabular}{|c|c|c|c|c|}
\hline Complications, $\mathrm{n}(\%)$ & $20(83.3)$ & $11(91.7)$ & $9(75.0)$ \\
\hline APGAR Scores & \multicolumn{3}{|c|}{} & $.847 \mathrm{a}$ \\
\hline 1 minute (median) $\mathrm{n}=23$ & 2 & 1.5 & 2 & $.433 \mathrm{a}$ \\
\hline 5 minute (median) $\mathrm{n}=23$ & 4 & 4 & 4 & $.582 \mathrm{a}$ \\
\hline 10 minute (median) $\mathrm{n}=20$ & 5 & 5 & 4.5 \\
\hline
\end{tabular}

andependent samples $t$-test

${ }^{\mathrm{b}}$ Chi square test

\section{Results and Discussion}

At the end of the data collection period, 18 of the 24 infants had undergone initial developmental testing with the BDI-II when they were between the ages of birth and 6 months. Of those evaluated, 11 infants were in the seizures group while 7 infants were in the no seizures group. In order to facilitate a comparison of our data with results of previously published studies, the total developmental quotients for each infant were described using standard categories of Average (85+), Moderately Delayed (70-84), and Severely Delayed $(<70)$.

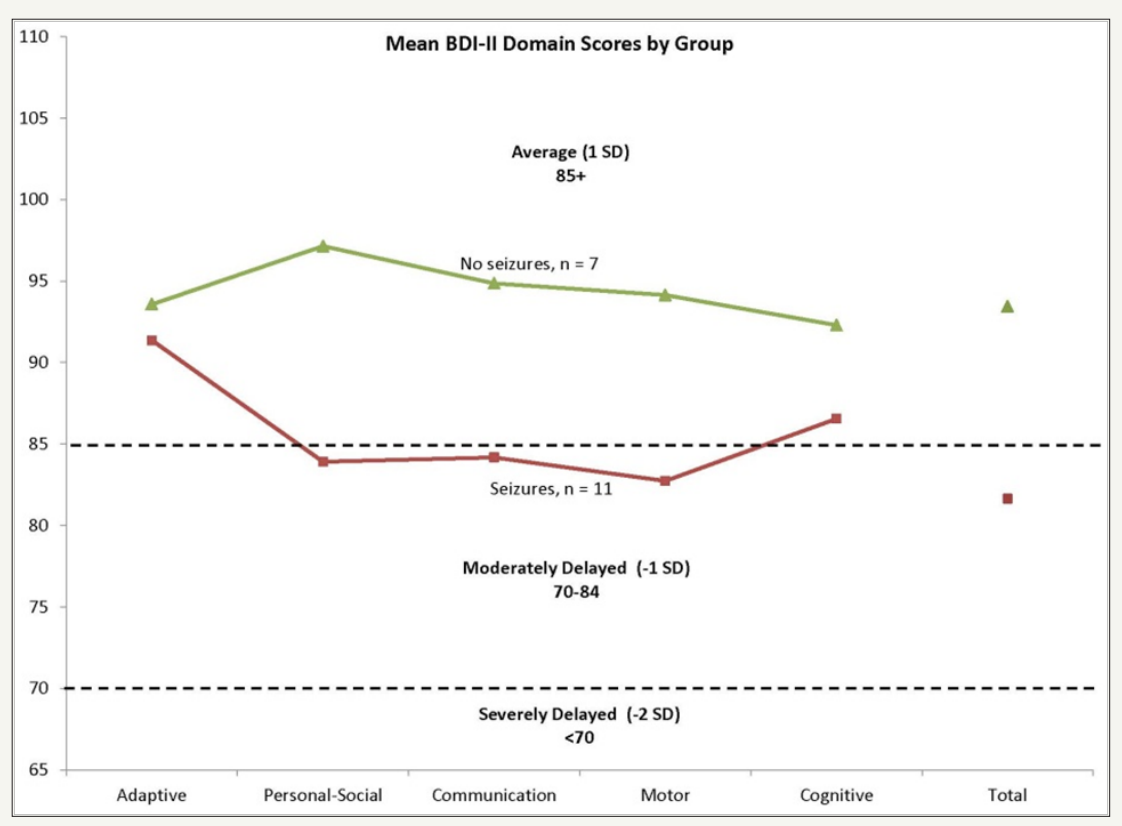

Figure 1: Mean standard scores for each domain on the BDI-II by group.

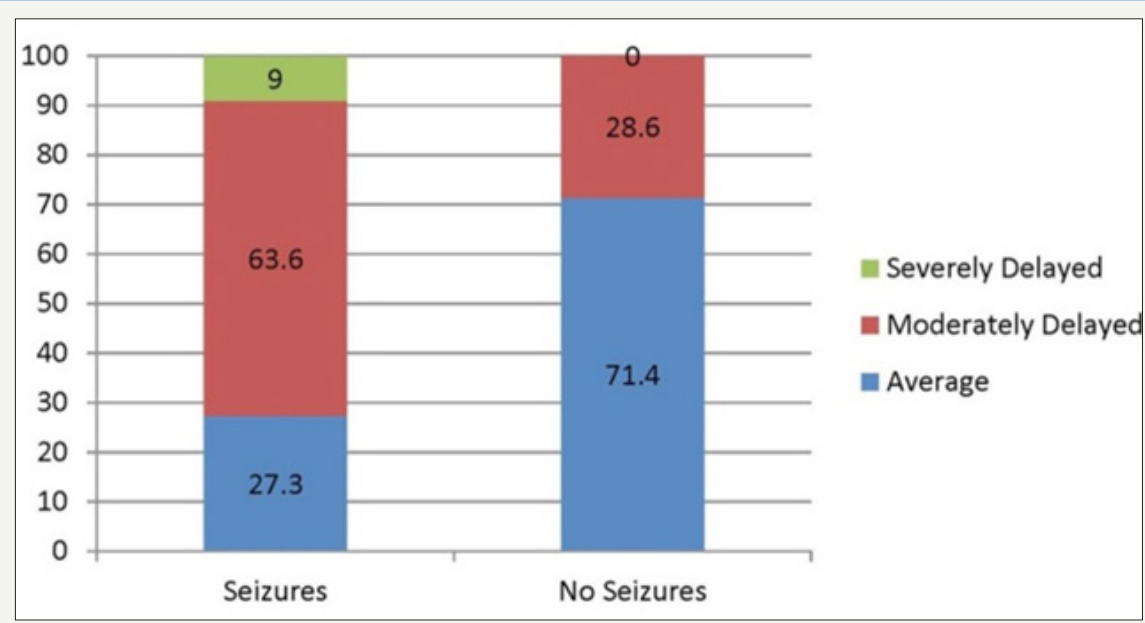

Figure 2: Percent of infants Total Developmental Quotient scores by descriptive category. 
Inconsistent with our first hypothesis, the majority of infants in this study did not achieve neurodevelopmental assessment scores within the Average range, which would have indicated comparable development to typically developing peers. Eleven infants scored in the Moderately Delayed or Severely Delayed ranges, while 7 infants scored within the Average range. Consistent with our second hypothesis, the group of infants without neonatal seizures had higher mean scores across all developmental domains than the group of infants with neonatal seizures; however, statistically significant differences were found only in the Personal-Social domain $[\mathrm{t}(15)=2.405, \mathrm{p}=.03]$ and the Total Developmental Quotient $[\mathrm{t}(15)=2.101, \mathrm{p}=.05]$. Refer to Figure 1 for a comparison of mean scores by domain area by subject group. Significantly more infants ( $\mathrm{p}=.03$ ) in our study who were in the no seizures group had total scores in the Average range $(71.4 \%)$ than did infants in the seizures group (27.3\%). Refer to Figure 2 for a comparison of the percent of scores in each descriptive category for the seizure and no seizure.

Previous studies [3] reported rates of approximately 69\% (Average), 7\% (Moderately Delayed), and 26\% (Severely Delayed) for infants who received a cooling treatment. Our study found different rates per category with considerably more infants falling in the Moderately Delayed range and fewer in the Severely Delayed range than previous studies (39\% Average, 55\% Moderately Delayed, 6\% Severely Delayed). In one of the few studies looking specifically at differences between infants with and without seizures, Kwon and colleagues (2011) found significantly higher rates of scores in the Severely Delayed range in the seizures group $(40 \%)$ than in the no seizures group (20\%), $p=02$. Our study found a similar pattern of scores with a significantly higher rate of scores in the Severely Delayed range for infants in the seizures group $(20 \%)$ than in the no seizures group $(0 \%)$. All of the infants in the no seizures group received developmental scores classified as Average or Moderately Delayed (none in the Significantly Delayed category).

\section{Conclusion}

Results of this study indicated that the majority of the infants had overall developmental scores that indicate Moderate to Severe Developmental Delays despite receiving the whole body cooling treatment. Specific comparisons between the group of infants with and without seizures who received this treatment indicated better developmental outcomes for infants without seizures than for those infants who had seizures, though statistically significant differences were found in one specific sub domain and in the overall Total Developmental Quotient. More of the infants in the no seizure group had developmental scores in the Average range than did infants in the seizures group $[4,5]$

\section{Impact \& Future Analysis}

The results of this clinical study enhanced our knowledge about the neurodevelopmental outcomes of therapeutic hypothermia for a specific cohort of infants and the possibility of differential outcomes given the presence or absence of seizure before, during, and after the cooling treatment. As a result of this study, hospitals may use the preliminary data to improve or modify the cooling protocol used in their NICU. Since the infants with seizures fared worse than those without seizures and clinical recognition of seizures in depressed and sedated babies remains challenging, earlier recognition of seizures utilizing Electroencephalogram (EEG) monitoring and earlier treatment for seizures may improve these infants' overall outcomes. This research data was shared with the administrators of the local early intervention program to assist the program in improving evaluation and intervention protocols.

As infants in the study reach appropriate ages for reevaluation, comparisons of the change in scores on the BDI-II will be made between infants with and without seizures. Infants are expected to be reevaluated at 18 months of age. Future evaluations are also recommended to evaluate executive functioning skills, attention and memory, and learning abilities, which previous research has indicated are skills and abilities negatively affected by prenatal and neonatal injuries such as HIE.

\section{References}

1. Rahman S (2011) Neuroprotection in neonatal hypoxic ischemic encephalopathy: Therapeutic hypothermia and beyond. Journal of Postgraduate Medical Institute 25(1): 1-3.

2. Garfinkle J, Shevell M (2011) Predictors of outcome in term infants with neonatal seizures subsequent to intrapartum asphyxia. J Child Neurol 26(4): 453-459.

3. Azzopardi D, Strohm B, Edwards A, Dyet L, Halliday H, et al. (2009) Moderate hypothermia to treat perinatal asphyxia encephalopathy. N Engl J Med 361(14): 1349-1358.

4. Kwon J, Guillet R, Shankaran S, Laptook A, McDonald S, et al. (2011) Clinical seizures in neonatal hypoxic-ischemic encephalopathy have no independent impact on neurodevelopmental outcome: Secondary analyses of data from the Neonatal Research Network Hypothermia Trial. J Child Neurol 26(3): 322-328.

5. Shah P, Ohlsson A, Perlman M (2007) Hypothermia to treat neonatal hypoxic ischemic encephalopathy: systematic review. Arch Pediatr Adolesc Med 161(10): 951-958.
Creative Commons Attribution 4.0 International License

For possible submissions Click Here

\section{Submit Article}

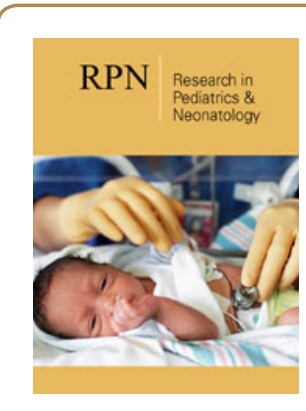

\section{Research in Pediatrics \& Neonatology}

\section{Benefits of Publishing with us}

- High-level peer review and editorial services

- Freely accessible online immediately upon publication

- Authors retain the copyright to their work

- Licensing it under a Creative Commons license

- Visibility through different online platforms 